\title{
Prevalence and determinants of use of complementary and alternative medicine by hypertensive patients attending primary health care facilities in Kinshasa, Democratic Republic of the Congo: a cross- sectional study
}

\author{
Aimée M. Lulebo ${ }^{1 *}$, Mala A. Mapatano ${ }^{1}$, Paulin B. Mutombo ${ }^{1}$, Eric M. Mafuta ${ }^{1}$, Gédéon Samba²
} and Yves Coppieters ${ }^{3}$

\begin{abstract}
Background: In the Democratic Republic of the Congo the control of hypertension is poor, characterized by an increasing number of reported cases of hypertension related complications. Poor control of hypertension is associated with non-adherence to antihypertensive medication. It is well established that the use of complementary and alternative medicine is one of the main factors of non-adherence to antihypertensive medication. The aim of this study is to determine the prevalence and factors associated with the use of complementary and alternative medicine.

Methods: A cross-sectional study was carried out at the Kinshasa Primary Health-care (KPHC) facilities network in November 2014. A structured interview questionnaire was administrated to a total of 280hypertensive patients. Complementary and alternative medicine were defined according to the National Institute of Health classification as a group of diverse medical and healthcare systems, practices, and products that are not presently considered to be part of conventional medicine. Data were summarized using proportion and mean (with standard deviation). The student's $t$ test and $x^{2}$ test were used respectively for mean and proportion comparison. Logistic regression analysis identified determinants of the use of complementary and alternative medicine.
\end{abstract}

Results: The prevalence of use of complementary and alternative medicine was $26.1 \%$ (95\% Cl: 20.7\% - 31.8\%). Determinants of use of complementary and alternative medicine included misperception about hypertension curability $(\mathrm{OR}=2.1 ; 95 \% \mathrm{Cl}: 1.1-3.7)$ and experience of medication side effects ( $\mathrm{OR}=2.9 ; 95 \% \mathrm{Cl}: 1.7-5.1)$.

Conclusion: The use of CAM for hypertensive patients is a major problem; antihypertensives with fewer side effects must be emphasized. Religious leaders must become involved in the communication for behavioral change activities to improve the quality of life for hypertensive patients.

Keywords: Hypertension, Complementary and alternative medicine, Medication adherence, DRC

\footnotetext{
* Correspondence: aimelulebo@yahoo.fr

${ }^{1}$ Department of Epidemiology and Biostatistics, Kinshasa School of Public

Health, Faculty of Medicine, University of Kinshasa, PO Box11850, Kinshasa1,

Democratic Republic of the Congo

Full list of author information is available at the end of the article
} 


\section{Background}

African countries are currently undergoing one of the most rapid epidemiological transitions. These countries have been characterized for a long time by incidence of infectious diseases, demographic and nutritional transitions contributing to a growing prevalence of noncommunicable diseases (NCDs) such as hypertension (HTN) [1, 2]. The number people with hypertension increased from 600 million in 1980 to 1 billion in 2008 [3]. HTN prevalence is higher in Africa (46\%) compared to America (35\%) [4]. In the Democratic Republic of the Congo (DRC), according to the World Health Organization (WHO), the prevalence of HTN was $32.1 \%$ in men and $31.5 \%$ in women in 2014 [5].

African countries are faced with the double burden of an increasing prevalence of HTN and other NCDs whilst simultaneously experiencing a high prevalence of infectious diseases that their Health Systems (HS) are unprepared to deal with [6,7]. The African population, accustomed to acute symptomatic infectious disease, is probably struggling to adapt to chronic diseases which are most of the time asymptomatic and requiring lifelong treatment.

Previous studies show that Africans were less adherent than Caucasians [8] to NCD treatment leading to poor control and higher incidence of complications. Uncontrolled HTN is one of the main cardiovascular risk factors (CVRF); it is one of the leading causes of premature death as it is associated with9.4 million deaths worldwide every year [9].

Non-adherence to antihypertensive medication (NAM) is an important factor of uncontrolled HTN [10]. In the DRC, the proportion of HTN control is very low with a high incidence of HTN-related complications including stroke, chronic kidney disease (CKD) [11-13]. A study carried out in Kinshasa in 2013 showed that only $15.6 \%$ of patients attending the primary health care facilities in Kinshasa had their blood pressure under control. Uncontrolled blood pressure was statistically associated with NAM. The same study also found that $20.5 \%$ of patients who took non-prescribed medication were twice more likely to be non-adherent to treatment than other patients [14]. This led us to believe that these hypertensive patients probably use CAM.

Adherence to treatment is mainly influenced by the perception of a patient towards the effectiveness of treatment and the quality of health care (its availability and affordability, and the relationship between patient and provider).In the event where care provided by a conventional health system is dissatisfactory, patients seem more likely to use alternative care currently known as complementary and alternative medicine (CAM) [15]. CAM is defined as: 'a group of diverse medical and healthcare systems, practices, and products that are not presently considered to be part of conventional medicine' [16].

This study was conducted to measure the prevalence of CAM and to identify its determinants. By understanding the predictors of CAM use, it could help to make place for interventions for improving treatment adherence and control of HTN.

\section{Methods}

\section{Study design}

In November 2014, a cross-sectional study was carried out at the Kinshasa Primary Health-care (KPHC) facilities network. This network consists of fifty-one facilities. It allows for follow-up of patients with NCDs such as hypertension and diabetes [14].

\section{Study population}

The study population included in the study were hypertensive patients $>18$ years old attending the KPHC facilities. Hypertensive pregnant women were excluded.

\section{Sampling}

Fifteen out of fifty-one health facilities organized patients' visits during the study period and were thus all included in the study. All patients present at the facility at the time of the visit and who met the inclusion criteria were enrolled. The sample size was computed using the following formula:

$$
n \geq \frac{Z_{\alpha}^{2} \cdot p \cdot q}{d^{2}}
$$

Where - $\mathbf{p}$ represents the proportion of hypertensive patients using CAM (we assumed a $p=19.5 \%$ ) $[17] ; \mathbf{q}(1-p) ; \mathbf{z}$ the value of the standard normal distribution coefficient corresponding to a significance level of alpha of 0.05 (1.96) and; $\mathbf{d}$ the precision degree that we assumed to $5 \%$ too. The minimal size computed was 246 patients.

\section{Data collection and study variables}

Face-to-face interviews using structured questionnaires were performed by six trained data collectors. The questionnaires were previously pretested with twenty-four patients who did not included in the analysis. The questionnaires were also translated into local language (Lingala) and back-translated in French before data collection. The following variables were collected:

- Demographic and social characteristics of patients (sex, age, educational level, religious affiliation, marital status, occupation); 
- Clinical characteristics of patients (duration of HTN, co-morbidity, experience of medication side effects, treatment adherence);

- Patient-related variables (knowledge of HTN risk factors and complications, knowledge of treatment benefit, perception of hypertension gravity, treatment effectiveness and HTN curability);

- Healthcare system variables (patient-provider relationship, waiting time, affordability and availability of healthcare); and

- CAM use description (use of CAM, CAM patterns, reasons for CAM use, information source about CAM).

To measure the use of CAM, the following questions were asked of the patient: "Do you currently use any method or substance other than those prescribed by your healthcare provider? If yes, what do you use?" A CAM user was defined as a patient who declared using one or more of the CAM categories in line with the definition provided by the National Institute of Health (NIH). NIH classifies CAM in 5 categories:

1) Alternative medical systems (e.g. traditional oriental medicine, acupuncture, Ayurveda, naturotherapy...).

2) Mind body intervention (meditation, hypnosis, dance, art, music-therapy, spiritual healing and prayer).

3) Biologically based therapies (herbal medicine and dietary supplements, special diets and orthomolecular medicine).

4) Manipulative and body based methods (chiropractic, massage...).

5) Energy therapies [16].

Comorbidity was defined in this study as HTN associated with Diabetes Mellitus (DM) or organ damage targets (heart disease, strokes, or CKD). Self-reported medication adherence was measured using the Morisky Medication Adherence Scale (MMAS), which is a validated scale with a good internal consistency [18, 19]. Patient responses of "yes" or "no" to the four questions were categorized into three groups, namely high, medium and low adherence, as summarized in Table 1. Thereafter, adherence was dichotomized in order to facilitate statistical analysis. Low and

Table 1 Morisky scale

High adherence, if the sum $=0$; medium adherence, if the sum is comprised between 1 and 2 and low adherence if the sum is comprised between 3 and 4 .

Have you ever forgotten to take your BP medicine? 0. No 1. Yes

Are you sometimes careless in regard to your medicine? 0. No 1. Yes

Do you skip your medicine when you are feeling well? $\quad 0$. No 1. Yes

When you feel bad due to the medicine, do you skip it? 0 . No 1. Yes

Source: Morisky et al. (1986). Concurrent and predictive validity of a self-reported measure of medication adherence.Med Care.1986; 24: 67-74 medium adherence was merged and classified as nonadherent and patients with high adherence were classified as adherent, as we have discussed previously [14].

Availability of healthcare facility (HCF) was determined by whether a HCF was available within a 5kilometerradius or 30 min walking distance from the patient. Affordability was defined by reference to the healthcare cost perception.

\section{Statistical analysis}

Stata version 12.0was used for statistical analysis. Descriptive statistics were used to summarize the characteristics of the study population. Continuous variables were reported using mean with standard deviation. Means for age and BP of CAM users and non-CAM users were compared using the Student's $t$ test. Categorical variables were reported as a frequency and percentage and groups were compared using the $x^{2}$ test. The forward stepwise logistic regression helped to identify independent predictors of CAM usage. All variables associated with CAM usage in the bivariate analysis were included in the final model. The odds ratio (OR) with a corresponding 95\% confidence interval was reported to quantify the strength of association. Significance was set at $p$-value of less than 0.05 .

\section{Results}

\section{Assessment of CAM use}

A total of 280 patients were interviewed. Tables 2 and 3 describe the CAM practices. Approximately a quarter of patients declared having used CAM, indicated by a prevalence of $26.1 \%$ (95\%CI: $20.7 \%$ - 31.8\%). Herbal medicines $(42.5 \%)$ and prayer $(35.6 \%)$ were the patterns of CAM use. The reasons mentioned by HTN patients for CAM use were effectiveness (34.2\%) and lower cost (28.8\%). Friends $(47.9 \%)$ and family members $(35.6 \%)$ were the sources of information on CAM.

\section{Demographic, social and clinical characteristics of participants}

Tables 4 and 5 summarize the socio-demographic and clinical characteristics of patients respectively. The mean age of participants was $60.1 \pm 10.6$ years and $68.6 \%$ were women; almost half $(52.5 \%)$ were married or cohabiting and $43.2 \%$ were Catholics; almost half (48.9\%) were unemployed and slightly more than half

Table 2 Frequency of CAM use

\begin{tabular}{ll}
\hline Variable $n=280$ & $N(\%)$ \\
\hline Use of CAM & \\
Yes & $73(26.1)$ \\
No & $207(73.9)$ \\
Total & $280(100.0)$ \\
\hline
\end{tabular}


Table 3 Description of CAM use

\begin{tabular}{ll}
\hline Variables $n=73$ & $N(\%)$ \\
\hline Patterns of CAM & $31(42.5)$ \\
Traditional medicine (self-medication/traditional healers) & $26(35.6)$ \\
Prayer & $13(17.8)$ \\
Chinese medicine & \\
Sources of information & $35(47.9)$ \\
Friends & $26(35.6)$ \\
Family members & $9(12.3)$ \\
Professional area & $8(11.0)$ \\
Media (radio, TV...) & \\
Reasons of CAM usage & $25(34.2)$ \\
Effectiveness of CAM & $21(28.8)$ \\
The low cost of CAM & $19(26.0)$ \\
Holistic care & $11(15.1)$ \\
Cultural reasons & $9(12.3)$ \\
CAM has little or no side effects &
\end{tabular}

(51.1\%) had completed at least secondary school. No statistical difference of socio-demographic characteristics was observed between CAM users and CAM non-users.

Moreover two-thirds (68.3\%) of participants suffered from HTN for less than 5 years and $56.4 \%$ experienced co morbidities, mainly diabetes. Almost onethird (39.3\%) of patients experienced side effects from medication; the CAM users declared experiencing more antihypertensive side effects than CAM nonusers $(60.3 \%$ vs $31.9 \%)(p=0,000)$. The use of the Morisky Scale showed that $74.7 \%$ of participants $(95 \%$ CI: $69.4 \%-79.9 \%)$ were non-adherent to their medication (NAM). However, NAM was not associated with the CAM use.

\section{Patients' knowledge and perception about HTN}

Table 6 summarizes the knowledge and perception of participants on HTN and its treatment. The majority of patients in both groups were unable to mention at least three risk factors of HTN (94.3\%) and HTN complications (94.3\%). Only $13.9 \%$ of the patients had good knowledge of the benefit of antihypertensive treatment. A lack of knowledge of the benefit of antihypertensive treatment was associated with CAM use $(p=0.022)$.

Table 4 Demographic and social characteristics of hypertensive patients using or not using CAM

\begin{tabular}{|c|c|c|c|c|}
\hline & Overall $n=280$ (\% or SD) & CAM users $n=73$ (\% or SD) & CAM non-users $n=207$ (\% or SD) & $p$ \\
\hline Mean age, years (SD) & $60.1 \pm 10.6$ & $58.7 \pm 9.8$ & $60.6 \pm 10.8$ & 0.190 \\
\hline \multicolumn{5}{|l|}{ Sex } \\
\hline Female & $192(68.6)$ & $50(68.5)$ & 142(68.6) & \multirow[t]{2}{*}{0.987} \\
\hline Male & $88(31.4)$ & $23(31.5)$ & 65 (31.4) & \\
\hline \multicolumn{5}{|l|}{ Religion } \\
\hline Catholic & $121(43.2)$ & $27(37.0)$ & $94(45.4)$ & \multirow[t]{6}{*}{0.287} \\
\hline Pentecostal & $57(20.4)$ & $20(27.4)$ & 37 (17.9) & \\
\hline Protestant & $48(17.1)$ & $9(12.3)$ & $39(18.8)$ & \\
\hline Kimbanguist & $22(7.9)$ & $7(9.6)$ & $15(7.3)$ & \\
\hline Jehovah's witnesses & $13(4.6)$ & $3(4.1)$ & $10(4.8)$ & \\
\hline Others & $19(6.8)$ & $7(9.6)$ & $12(5.8)$ & \\
\hline \multicolumn{5}{|l|}{ Marital status } \\
\hline Married/cohabiting & $147(52.5)$ & $41(56.2)$ & 106(51.2) & \multirow[t]{2}{*}{0.466} \\
\hline Single/separated/divorced/widowed & $133(47.5)$ & $32(43.8)$ & $101(48.8)$ & \\
\hline \multicolumn{5}{|l|}{ Occupation } \\
\hline No & $137(48.9)$ & $41(56.2)$ & $96(46.4)$ & \multirow[t]{2}{*}{0.150} \\
\hline Yes & $143(51.1)$ & $32(43.8)$ & 111 (53.6) & \\
\hline \multicolumn{5}{|l|}{ Educational level } \\
\hline Primary & $85(30.4)$ & $18(24.7)$ & 67 (32.4) & \multirow[t]{3}{*}{0.213} \\
\hline Secondary & $144(51.4)$ & $44(60.3)$ & $100(48.3)$ & \\
\hline Post-secondary & $51(18.2)$ & $11(15.0)$ & 40 (19.3) & \\
\hline
\end{tabular}


Table 5 Clinical characteristics of hypertensive patients using or not using CAM

\begin{tabular}{|c|c|c|c|c|}
\hline & Overall $n=280$ (\% or SD) & CAM users $n=73$ (\% or SD) & CAM non-users $n=207$ (\% or SD) & $p$ \\
\hline \multicolumn{5}{|c|}{ Duration of HTN } \\
\hline$\geq 5$ years & 89 (31.8) & $23(31.5)$ & $66(31.9)$ & \multirow[t]{2}{*}{0,953} \\
\hline$<5$ years & $191(68.2)$ & $50(68.5)$ & $141(68.1)$ & \\
\hline \multicolumn{5}{|l|}{ Co morbidity } \\
\hline Yes & $158(56.4)$ & $37(50.7)$ & $121(58.5)$ & \multirow[t]{2}{*}{0,249} \\
\hline No & $122(43.6)$ & $36(49.3)$ & $86(41.5)$ & \\
\hline \multicolumn{5}{|c|}{ Experiencing side effects } \\
\hline Yes & $110(39.3)$ & $44(60.3)$ & $66(31.9)$ & \multirow[t]{2}{*}{$0,000^{\mathrm{a}}$} \\
\hline No & $170(60.7)$ & $29(39.7)$ & $141(68.1)$ & \\
\hline \multicolumn{5}{|c|}{ Treatment adherent $(n=265)$} \\
\hline No & 198 (74.7) & $76(71.7)$ & $122(76.7)$ & 0.356 \\
\hline
\end{tabular}

${ }^{\mathrm{a} S t a t i s t i c a l l y ~ s i g n i f i c a n t ~}$

Most patients (82.4\%) knew that HTN was a serious health problem. Close on two-thirds (61.4\%) of patients thought of HTN as curable. This misperception of HTN curability was statistically associated with CAM use $(p=0.045)$.

\section{Healthcare system factors}

Table 7 summarizes the health team and system variables. The study shows that the majority of patients declared that healthcare facilities were near their households (87.9\%). Appreciation of the relationship with health care providers (good or very good) was almost universal (96.1\%) although nearly half of the patients $(53.6 \%)$ acknowledged that they had to wait more than $30 \mathrm{~min}$ before receiving service. The long waiting time was associated with CAM use $(p=0.015)$. Two-thirds of the patients thought that the cost of treatment provided at healthcare facilities was affordable (65.0\%). The majority of the patients declared that they were globally satisfied with the quality of healthcare (86.8\%).

\section{Determinants of CAM use}

Table 8 summarizes the determinants of CAM use with bivariate and multivariate analysis. Bivariate analysis shows that the lack of knowledge of treatment benefits by patients $(\mathrm{OR}=2.3$; 95\% CI: 1.1-4.8), the experiencing of side effect from medication $(\mathrm{OR}=3.2 ; 95 \% \mathrm{CI}: 1.8-5.9)$ and the long waiting time $(\mathrm{OR}=2.0 ; 95 \% \mathrm{CI}$ : $1.1-3.6)$ were associated with CAM use. The misperception of

Table 6 Use of CAM,knowledge and perception of HTN

\begin{tabular}{|c|c|c|c|c|}
\hline & Overall $n=280$ (\% or SD) & CAM users $n=73$ (\% or SD) & CAM non-users $n=207$ (\% or SD) & $p$ \\
\hline \multicolumn{5}{|l|}{ Knowledge of HTN risk factors } \\
\hline Have cited a least three & $16(5.7)$ & $3(4.1)$ & $13(6.3)$ & \multirow[t]{2}{*}{0.492} \\
\hline Have cited less than three & $264(94.3)$ & $70(95.9)$ & $194(93.7)$ & \\
\hline \multicolumn{5}{|l|}{ Knowledge of HTN complications } \\
\hline Have cited a least three & $16(5.7)$ & $1(1.4)$ & $15(7.2)$ & \multirow[t]{2}{*}{0.063} \\
\hline Have cited less than three & $264(94.3)$ & $72(98.6)$ & $192(92.8)$ & \\
\hline \multicolumn{5}{|l|}{ Knowledge of treatment benefits } \\
\hline Have cited less than 3 & $241(86.1 \%)$ & 57 (78.1\%) & $184(88.9 \%)$ & \multirow[t]{2}{*}{$0,022^{\mathrm{a}}$} \\
\hline Have cited a least three & 39 (13.9\%) & $16(21.9 \%)$ & $23(11.1 \%)$ & \\
\hline \multicolumn{5}{|l|}{ Perception of HTN gravity } \\
\hline HTN is not a serious health condition & $49(17.5 \%)$ & $14(19.2 \%)$ & 35 (16.9\%) & \multirow[t]{2}{*}{0,673} \\
\hline HTN is a serious health condition & $231(82.5 \%)$ & $59(80.8 \%)$ & $172(83.1 \%)$ & \\
\hline \multicolumn{5}{|l|}{ Perception HTN curability } \\
\hline HTN is curable & $172(61.4 \%)$ & $52(71.2 \%)$ & $120(58.0 \%)$ & \multirow[t]{2}{*}{$0,045^{a}$} \\
\hline HTN is not curable & $108(38.6)$ & $21(28.8 \%)$ & 87 (42.0\%) & \\
\hline
\end{tabular}


Table 7 Healthcare system factors

\begin{tabular}{|c|c|c|c|c|}
\hline & Overall $n=280$ (\% or SD) & CAM users $n=73$ (\% or SD) & CAM non-users $n=207$ (\% or SD) & $p$ \\
\hline \multicolumn{5}{|l|}{ Availability of HCF } \\
\hline Not available & $34(12.1)$ & $7(9.6)$ & $27(13.0)$ & \multirow[t]{2}{*}{0.437} \\
\hline Available & $246(87.9)$ & $66(90.4)$ & 180(87.0) & \\
\hline \multicolumn{5}{|l|}{ Waiting time } \\
\hline Very long/long & $150(53.6)$ & $48(65.8)$ & $102(49.3)$ & \multirow[t]{2}{*}{$0.015^{\mathrm{a}}$} \\
\hline Very short/short & $130(46.4)$ & $25(34.2)$ & 105(50.7) & \\
\hline \multicolumn{5}{|l|}{ Relationship } \\
\hline Somewhat good/bad & $11(3.9)$ & $3(4.1)$ & $8(3.9)$ & \multirow[t]{2}{*}{0.581} \\
\hline Very good/good & $269(96.1)$ & 70 (95.9) & $199(96.1)$ & \\
\hline \multicolumn{5}{|l|}{ Affordability } \\
\hline Not affordable & $98(35.0)$ & $26(35.6)$ & $72(34.8)$ & \multirow[t]{2}{*}{0.898} \\
\hline Affordable & $182(65.0)$ & $47(64.4)$ & $135(65.2)$ & \\
\hline \multicolumn{5}{|l|}{ Global satisfaction } \\
\hline No & $37(13.2)$ & $11(15.1)$ & $26(12.6)$ & \multirow[t]{2}{*}{0.586} \\
\hline Yes & $243(86.8)$ & $62(84.9)$ & $181(87.4)$ & \\
\hline
\end{tabular}

${ }^{\mathrm{a}}$ Statistically significant

HTN curability $(\mathrm{OR}=1.8 ; 95 \% \mathrm{CI}$ : $0.97-3.3)$ has been also included in the multivariate analysis because its $p$ value (0.045). Multivariate analysis identified a misperception by patients of HTN curability $(\mathrm{OR}=2.1$; 95\%CI: 1.1-3.7) and experiencing of side effects from medication $(\mathrm{OR}=2.9 ; 95 \% \mathrm{CI}: 1.7-5.1)$ as predictors of CAM use.

Patient's characteristics and perception of HTN curability Table 9 shows that the duration of HTN $(p=0.022)$ and religious affiliation (0.006) were statistically associated with the misperception of HTN curability.

\section{Discussion}

The study found that more than a quarter of patients used CAM (26.1\%); mainly the biologically based therapies and the mind-body interventions.

The misperception of HTN curability and experience of medication side effects were independent determinants of CAM use. Religious affiliation and HTN duration were associated with misperception of HTN curability.

The present study confirmed our hypothesis asserted in a study conducted previously that a sizeable proportion of patients with hypertension in Kinshasa use

Table $\mathbf{8}$ Bivariate and multivariate analysis determinants of CAM use

\begin{tabular}{|c|c|c|c|c|c|c|}
\hline \multirow[t]{3}{*}{ Factors } & \multicolumn{6}{|l|}{ CAM use } \\
\hline & \multicolumn{3}{|c|}{ Bivariate analysis } & \multicolumn{3}{|c|}{ Multivariate analysis (LR) } \\
\hline & Crude OR & $(95 \% \mathrm{Cl})$ & $p$ & Adjusted OR & $(95 \% \mathrm{Cl})$ & $p$ \\
\hline \multicolumn{7}{|c|}{ Knowledge of treatment benefits } \\
\hline Have cited less than 3 & 2.3 & $(1.1-4.8)$ & $0.022^{\mathrm{a}}$ & & & \\
\hline Have cited a least 3 & 1 & - & - & - & - & - \\
\hline \multicolumn{7}{|l|}{ Perception of HTN curability } \\
\hline HTN is curable & 1.8 & $(0.97-3.3)$ & $0.045^{a}$ & 2.1 & $(1.1-3.7)$ & $0.019^{a}$ \\
\hline HTN is incurable & 1 & - & - & - & - & - \\
\hline \multicolumn{7}{|l|}{ Experiencing side effects } \\
\hline Yes & 3.2 & $(1.8-5.9)$ & $0.000^{\mathrm{a}}$ & 2.9 & $(1.7-5.1)$ & $0.000^{\mathrm{a}}$ \\
\hline No & 1 & - & - & - & - & - \\
\hline \multicolumn{7}{|l|}{ Waiting time } \\
\hline Very long/long & 2.0 & $(1,1-3.6)$ & $0,015^{\mathrm{a}}$ & & & \\
\hline Very short/short & 1 & - & - & - & - & - \\
\hline
\end{tabular}

${ }^{a}$ Statistically significant 
Table 9 The perception of HTN curability and patient's characteristics

\begin{tabular}{|c|c|c|c|c|}
\hline & \multirow[b]{2}{*}{ Overall $(n=280)$} & \multicolumn{3}{|c|}{ Perception of HTN curability } \\
\hline & & HTN is curable $(n=172)$ & HTN is incurable $(n=108)$ & $p$ \\
\hline Mean age, years (SD) & $60.1(10.6)$ & $60.2(11.0)$ & $60.0(9.8)$ & 0.879 \\
\hline \multicolumn{5}{|l|}{ Sex } \\
\hline Male & $88(31.4)$ & $54(61.4)$ & 34 (38.6) & \multirow[t]{2}{*}{0.988} \\
\hline Female & $192(68.6)$ & $118(61.5)$ & $74(38.5)$ & \\
\hline \multicolumn{5}{|l|}{ Educational level } \\
\hline Primary & $85(30.6)$ & $54(63.5)$ & $31(36.5)$ & \multirow[t]{3}{*}{0.691} \\
\hline Secondary & $142(51.1)$ & $85(59.0)$ & $59(41.0)$ & \\
\hline Post-secondary & $51(18.3)$ & $33(64.7)$ & $18(35.3)$ & \\
\hline \multicolumn{5}{|l|}{ Religious affiliation } \\
\hline Catholic & $121(43.2)$ & $65(53.7)$ & $56(46.3)$ & \multirow[t]{6}{*}{$0.006^{\mathrm{a}}$} \\
\hline Pentecostal & $57(20.4)$ & $46(80.7)$ & $11(19.3)$ & \\
\hline Protestant & $48(17.1)$ & $29(60.4)$ & 19(39.6) & \\
\hline Kimbanguist & $22(7.9)$ & $16(72.7)$ & $6(27.3)$ & \\
\hline Jehovah's witnesses & $13(4.6)$ & $5(38.5)$ & $8(61.5)$ & \\
\hline Others & $19(6.8)$ & $11(57.9)$ & $8(42.1)$ & \\
\hline \multicolumn{5}{|l|}{ Duration of HTN } \\
\hline$\geq 5$ years & 89 (31.8) & $46(51.7)$ & $43(48.3)$ & \multirow[t]{2}{*}{$0.022^{a}$} \\
\hline$<5$ years & $191(68.2)$ & $126(66.0)$ & 65(34.0) & \\
\hline
\end{tabular}

${ }^{\mathrm{a} S t a t i s t i c a l l y ~ s i g n i f i c a n t}$

alternative care [14].The proportion of the use of CAM found in this study $26.1 \%$ (95\% CI $20.7 \%-31.8 \%)$ is similar to that found by previous studies conducted in sub-Saharan Africa (SSA) [20, 21].

Alternative treatments used by the majority of respondents included medicinal plants by self-medication or prescribed by herbalists, followed by prayer. These findings are similar to those found in previous studies $[15,22]$. This can be explained in the current context of the city of Kinshasa where so many commercial traditional healers promote the values of their plants, pretending that they can cure even incurable diseases such HTN, and DM. Amira and Okubadejo in Nigeria observed that powerful advertisements placed by alternative medicine practitioners encouraged patients to use as a remedy for all diseases, a panacea [23]. Thus, they advocate healing without regard to the observance of certain dietary measures that are described as an important factor affecting the quality of life of patients.

The health expectations of patients are linked to adherence to conventional treatment and to use of CAM. The CAM practitioners interviewed during a qualitative study carried out in Nigeria declared that the main reason patients consulted them was the belief that CAM practitioners could cure HTN at a more reasonable cost than the HCF [23]. The perceived effectiveness and low cost were the main reasons for the use of CAM cited in this study. The study found that patients who believed that hypertension is curable were more likely to use CAM.

Literature indicates that in developing countries, the population has a strong belief in the efficacy of natural and local resources in treating diseases. These resources are described by people as having no side effects unlike modern medicine [17]. This study also found that the presence of side effects of medications was independently associated with the use of alternative care; this result is consistent with earlier studies [17, 24-26].

Healthy behavior models increasingly describe the impact of social determinants such as spirituality and religion on health and treatment adherence [27]. Spirituality and religion can lead to erroneous beliefs that can lead to the denial of the disease which is a determinant of non-adherence [28]. Spiritual and religious beliefs can therefore have a significant effect on health behavior. The study found that more than two thirds of hypertensive patients thought they can cure their disease and this misperception was a factor in the use of alternative medicine. The misperception of HTN curability was associated with religious affiliation, majority of patients of Pentecostal churches declared that HTN is curable comparatively to others religious affiliation. For more than a decade, there has been an influx of Pentecostal churches in Kinshasa that have brought a new current of thought of all fields of life of the populace. These churches are authors of miracle cures and advocate the supremacy of 
God who heals; to accept an incurable chronic disease would be to ignore the power of God. Religious leaders are important authority figures for the population of Kinshasa and the former could constitute an important target for communication on behavioral change.

The study did not find any association between NAM and CAM use. This can be explained by the tool used for measuring NAM -the MMAS, comprising of four questions, which probably failed to detect that some people can refuse to take their medicine because they believe that HTN can be cured by God.

The study was conducted in hospitals and thus probably underestimated the frequency of the use of alternative care. Furthermore NAM was measured using a self-reported questionnaire. Nevertheless, this study is one of the first to have described this problem in the DRC and the results found are consistent with those described in the literature.

\section{Conclusion}

The use of CAM for hypertensive patients attending the primary healthcare facilities in Kinshasa is a major problem; antihypertensives with fewer side effects must be emphasized and religious leaders must become involved in the communication and advocation of behavioral change in order to improve the quality of life of hypertensive patients.

\section{Additional file}

Additional file 1: Dataset. This file contains data supporting the results of this study. (DTA $405 \mathrm{~kb}$ )

\begin{abstract}
Abbreviations
BP: Blood pressure; CAM: Complementary and Alternative Medicine; CKD: Chronic kidney disease; CVD: Cardiovascular diseases;

CVRF: Cardiovascular risk factors; DM: Diabetes Mellitus; DRC: Democratic Republic of Congo; HCF: Healthcare Facility; HS: Health System; HTN: Hypertension; KPHC: Kinshasa Primary Health-care; MMAS: Morisky Medication Adherence Scale; NAM: Non-adherence to antihypertensive medication; NCDs: Non communicable diseases; NHI: National Institute of Health; OR: Odds Ratio; SSA: Sub-Saharan Africa; WHO: World Health Organization
\end{abstract}

\section{Acknowledgements}

We acknowledge the Bureau Diocésain des Oeuvres Médicales de Kinshasa (BDOM) and the Bureau Médical de l'Armée du Salut for their approval of data collection in their health care facilities. We acknowledge, too, all health care providers and patients for their participation.

\section{Funding}

We are thankful to the United States Agency for International Development (USAID) for their funding of data collection. Also, we are grateful to Future Health Systems (FHS) for providing small grants to the Kinshasa School of Public Health for master students working on Health systems related topics through the Africa Hub, funded by the Department for International Development (DFID). These funding sources are non-commercial.

\section{Availability of data and materials}

The data set supporting the results of this study is available in an Additional file 1. This data set is labeled in French.

\section{Authors' contributions}

GS, AL and PM designed the research, AL and PM conducted the fieldwork and analyzed data; AL, PM and EM wrote the paper; and M M and YC revised the manuscript. AL has the primary responsibility for the final content. All authors read and approved the final manuscript.

\section{Competing interests}

The authors declare that they have no competing interest.

\section{Consent for publication}

Written informed consent was obtained from all participants to publish information coming from the survey.

\section{Ethics approval and consent to participate}

The study protocol was approved by the institutional review board of the Kinshasa School of Public Health (n ESPND/DIR/TK/214/KL/2014). This study was conducted in accordance with the Helsinki Declaration II. All study participants provided written informed consent.

To ensure patient confidentiality, the exit interviews were conducted in the absence of the healthcare provider and for anonymity, neither the name nor the patient's address were collected.

\section{Publisher's note}

Springer Nature remains neutral with regard to jurisdictional claims in published maps and institutional affiliations.

\section{Author details}

${ }^{1}$ Department of Epidemiology and Biostatistics, Kinshasa School of Public Health, Faculty of Medicine, University of Kinshasa, PO Box11850, Kinshasa1, Democratic Republic of the Congo. ${ }^{2}$ Programme National de lutte contre les maladies tropicales négligées, Kinshasa, Democratic Republic of the Congo. ${ }^{3}$ School of Public Health, Université Libre de Bruxelles (ULB), Bruxelles, Belgium.

Received: 23 September 2016 Accepted: 4 April 2017

Published online: 08 April 2017

\section{References}

1. Njelekela MA, Mpembeni R, Mligiliche NL, Spiegelman D, Hertzmark E, Liu E, etal. Gender related differences intheprevalenceofcardiovasculardis easeriskfactorsandtheircorrelatedin urbanTanzania. BMC. 2009;17:9-30.

2. YusufS RS, Ôunpuu S, Anand S. Globalburden ofcardiovasculardiseasespartl: general considerations, theEpidemiologicTransition, RiskFactors, and ImpactofUrbanization. Circulation. 2001;104:2746-53.

3. World Health Organization. Global status report on non-communicable diseases in 2010. Geneva: World Health Organization; 2011.

4. World Health Organization. Global Health Observatory Data Repository. Geneva: World Health Organization; 2008.

5. OMS, Genève. World health statistics 2015. Available at: http://www.who. int/mediacentre/news/releases/2015/world-health-statistics-2015/fr/ . Accessed 03 aug 2015

6. Belue R, Okoror TA, Lwelunmor J, Taylor KD, Degboe AN, Agyemang C, et al. An overview of cardiovascular risk factor burden in sub-Saharan African countries: a socio-cultural perspective. Global Health. 2009;22:5-10.

7. Mamo Y, Seid E, Adams S, Gardiner A, Parry E. A primary health care approach to the management of chronic diseases in Ethiopia: an example for other countries. Clin Med. 2007:7:228-31.

8. Osterberg L, Blaschke T. Adherence to medication. N Engl J Med. 2005; 353(5):487-97.

9. Lim SS, Vos T, Flaxman AD, Danaei G, et al. A comparative risk assessment of burden of diseases and injury attributable to 67 risk factors and risk factor clusters in 21 regions, 1990-2010: a systematic analysis for the global burden of disease study 2010. Lancet. 2012;380(9859):2224-60.

10. Matsumura K, Arima H, Tominaga M, Ohtsubo T, Sasaguri T, Fuji K, et al. Impact of antihypertensive medication adherence on blood pressure control in hypertension: the COMFORT study. Q J Med. 2013;106:909-14.

11. Sumaili EK, Krzesinski JM, Cohen EP, Nseka NM. Epidemiologie de la maladie rénale chronique en Republique Démocratique du Congo : revue des études transversales de Kinshasa, la capitale. NephrolTher. 2010;6(4):232-9. 
12. Sumaili KE, Nseka NM, Makulo JRR, Zinga CV, Longo AL, Mukendi SK et al. Statut socioéconomique et protéinurie: résultats de campagne de dépistage des maladies des reins 2007 à Kinshasa. Ann Afr Med. 2009; $2 n^{\circ} 3$.

13. M'Buyamba KJR, Biswika RT, Thijs L, Tshimanga GM, Ngalula FM, Disashi T et al. In-hospital mortality among black patients admitted for hypertensionrelated disorders in Mbuji Mayi, Congo. Am J Hypertens. 2009; 22: 643-648.

14. Lulebo MA, Mutombo BP, Mapatano MA, Mafuta ME, Kayembe KP, Ntumba TL, Mayindu NA, Coppieters Y. Predictors of non-adherence to antihypertensive medication in Kinshasa, Democratic Republic of Congo: A cross-sectional study BMC Res Notes. 2015; 8:526

15. Hughes GD, Oluwaseyi MA, Bobby LC, Thandi RP. The prevalence of traditional herbal medicine use among hypertensives living in South African communities, BMC Complementary and Alternative Medicine. 2013.

16. NCCAM (National Center for Complementary and Alternative Medicine). Available at: http://nccam.nih.gov/health/whatiscam/. Accessed 13 Mar 2015.

17. Kretchy Al, Owusu-Daaku F, Danquah S. Patterns and determinants of the use of complementary and alternative medicine: a cross-sectional study of hypertensive patients in Ghana. BMC Complement Altern Med. 2014;14:44.

18. Morisky DE, Green LW, Levine DM. Concurrent and predictive validity of a self-reported measure of medication adherence. Med Care. 1986;24:67-74.

19. Shea S, Misera D, Ehrlich MA. Correlates of non-adherence to hypertension treatment in an inner-city minority population. Am J Public Health. 1992;82:1607-12.

20. Osamor PE, Owumi BE. Complementary and alternative medicine in the management of hypertension in an urban Nigerian community. BMC Complement Altern Med. 2010;10:36.

21. Nuwaha F, Musinguzi G. Use of alternative medicine for hypertension in Buikwe and Mukono districts of Uganda: a cross sectional study. BMC Complement Altern Med. 2013;13:301.

22. $\mathrm{Hu} \mathrm{H}, \mathrm{Li}$ G, Duan J, Arao T, Prevalence, Purposes, and Perceived Effectiveness of Complementary and Alternative Medicine Use in a Hypertension Population: A Questionnaire Survey, Hindawi Publishing Corporation Volume 2013, Article ID 137472, 7 pages.

23. Amira Oluwatoyin C, OkubadejoNjideka U. Frequency of complementary and alternative medicine utilization in hypertensive patients attending an urban tertiary care Centre in Nigeria. BMC Complement Altern Med. 2007;7:30

24. Delgoda R, Pickinga D, Youngerb N, Mitchellc S. The prevalence of herbal medicine home use and concomitant use with pharmaceutical medicines in Jamaica. J Ethnopharmacol. 137 (2011) 305- 311.

25. Mollaoğlu M, Aciyurt A. Use of complementary and alternative medicine among patients with chronic diseases. Acta Clin Croat. 2013; 52:181-188.

26. Ching SM, Ramachandran V, Zainul AZ, Fuziah P. Frequency of complementary and alternative medicine usage among Malaysian hypertensive subjects. Life Sci J. 2013;10(4):2526-31.

27. Kretchy Al, Owusu-Daaku F, Danquah S. Spiritual and religious beliefs: do they matter in medication adherence behavior of hypertensive patients? Biopsych Med. 2013;7:15.

28. Komi Agboli. l'impact des maladies chroniques sur l'utilisation des services de santé, université du QUEBEC à Montréal; 2012.

\section{Submit your next manuscript to BioMed Central and we will help you at every step:}

- We accept pre-submission inquiries

- Our selector tool helps you to find the most relevant journal

- We provide round the clock customer support

- Convenient online submission

- Thorough peer review

- Inclusion in PubMed and all major indexing services

- Maximum visibility for your research

Submit your manuscript at www.biomedcentral.com/submit
Biomed Central 